\title{
Measuring Tail Dependence for Aggregate Collateral Losses Using Bivariate Compound Shot-Noise Cox Process
}

\author{
Jiwook Jang ${ }^{1}$, Genyuan Fu ${ }^{2}$ \\ ${ }^{1}$ Department of Applied Finance \& Actuarial Studies, Faculty of Business and Economics, \\ Macquarie University, Sydney, Australia \\ ${ }^{2}$ Benelec Pty Ltd., Sydney, Australia \\ Email: jiwook.jang@mq.edu.au, paulg@benelec.com.au
}

Received October 4, 2012; revised November 4, 2012; accepted November 11, 2012

\begin{abstract}
In this paper, we introduce tail dependene measures for collateral losses from catastrophic events. To calculate these measures, we use bivariate compound process where a Cox process with shot noise intensity is used to count collateral losses. A homogeneous Poisson process is also examined as its counterpart for the case where the catastrophic loss frequency rate is deterministic. Joint Laplace transform of the distribution of the aggregate collateral losses is derived and joint Fast Fourier transform is used to obtain the joint distributions of aggregate collateral losses. For numerical illustrations, a member of Farlie-Gumbel-Morgenstern copula with exponential margins is used. The figures of the joint distributions of collateral losses, their contours and numerical calculations of risk measures are also provided.
\end{abstract}

Keywords: Aggregate Collateral Losses; Bivariate Compound Cox Process; Shot Noise Process; Farlie-Gumbel-Morgenstern Copula; Tail Dependence; Joint Fast Fourier Transform

\section{Introduction}

Over the recent years, numerous papers have looked at the modelling of dependence within an insurance portfolio or between insurance portfolios [1-5]. Also in the field of financial risk management, a range of papers on dependence modelling within credit risk and operational risk can be noticed [6-8]. Besides the construction of specific multivariate models, considerable attraction is given to the use of copulas. In particular, within the theory of Lévy processes, Lévy copulas have proven to be useful [9].

Our paper is very much based on insurance applications where two lines of business are hit by a common external event, hence the word "collateral losses" in the title. These joint losses may, for instance be triggered by events such as flood, windstorm, hail, bushfire, earthquake and terrorism. Particular examples concern collateral losses due to 2011 Great Eastern Japan Earthquake, 2010-2011 Queensland floods, 2009 Victorian Bushfires [10], 2005 Hurricane Katrina [11] and 2001 September 11 attack [12].

For the purpose of this paper, we concentrate on a very specific model and show how, within this model several explicit calculations for relevant risk quantities can be performed. The bivariate model we consider has the following structure:

$$
L_{t}^{(1)}=\sum_{i=1}^{N_{t}} X_{i}, L_{t}^{(2)}=\sum_{i=1}^{N_{t}} Y_{i},
$$

where $L_{t}^{(k)}$ is the total loss arising from risk type $k=1,2$ and $N_{t}$ is the number of collateral losses up to time $t$. The random variables $X_{i}$ and $Y_{i}, i=1,2, \cdots$, denote the individual loss amounts. In this model, the dependence between two random variables $L_{t}^{(1)}$ and $L_{t}^{(2)}$ comes from the common arrival process $N_{t}$, together with the dependence between the individual losses $X_{i}$ and $Y_{i}$. The latter is modelled using the notion of copula [13]. To be more specific, we assume the loss random variable $X_{i}$ and $Y_{i}$ are independent identically distributed with continuous distribution function $F_{X}$ and $F_{Y}$ respectively. The joint distribution of the vector $(X, Y)$ is assumed to be of the form $C\left(F_{X}, F_{Y}\right)$ with a given copula $C$. The uniqueness of this two stage construction goes back to Sklar's Theorem.

Theorem 1.1. (Sklar's Theorem) Let $F$ be a joint distribution function with margins $F_{X}$ and $F_{Y}$. Then there exists a copula $C$ such that for all $x, y$ in $\overline{\mathbb{R}}=[-\infty, \infty]$,

$$
F(x, y)=C\left(F_{X}(x), F_{Y}(y)\right) .
$$


If the margins are continuous, then $C$ is unique; otherwise $C$ is uniquely determined on $\operatorname{Ran} F_{X} \times \operatorname{Ran} F_{Y}$, where $\operatorname{Ran} F_{i}=F_{i}(\overline{\mathbb{R}})$ denotes the range of $F_{i}$. Conversely, if $C$ is a copula and $F_{X}$ and $F_{Y}$ are univariate distribution functions, then the function $F$ defined in (2) is a joint distribution function with margins $F_{X}$ and $F_{Y}$.

Proof. See Schweizer and Sklar [14] or Nelsen ([13], p. 18).

To deal with stochastic nature of catastrophic loss arrival in practice, we use a Cox process for $N_{t}$. The Cox process provides flexibility by letting the intensity not only depend on time but also allowing it to be a stochastic process. Therefore the Cox process can be viewed as a two step randomisation procedure. A process $\lambda_{t}$ is used to generate another process $N_{t}$ by acting its intensity. That is, $N_{t}$ is a Poisson process conditional on $\lambda_{t}$ which itself is a stochastic process.

Losses arising from a catastrophe depend on its intensity. One of the processes that can be used to measure the impact of catastrophic events is the shot noise process. Previous works of insurance application using shot noise process and a Cox process with shot noise intensity can be found in [15-19]. Reference [20] also used a Cox process with shot noise intensity to model operational risk. The shot noise process is particularly useful to loss arrival process as it measures the frequency, magnitude and time period needed to determine the effect of catastrophic events. As time passes, the shot noise process decreases as more and more losses are settled. This decrease continues until another event occurs which will result in a positive jump in the shot noise process. Therefore the shot noise process can be used as the parameter of a Cox process to measure the number of catastrophic losses, i.e. we will use it as an intensity function to generate a Cox process. We will adopt the shot noise process used by Cox \& Isham [21]:

$$
\lambda_{t}=\lambda_{0} \mathrm{e}^{-\delta t}+\sum_{i=1}^{M_{t}} Z_{i} \mathrm{e}^{-\delta\left(t-S_{i}\right)}
$$

where:

- $\lambda_{0}$ is the initial value of $\lambda_{t}$ that is carried on from catastrophic events incurred previously;

- $\left\{Z_{i}\right\}_{i=1,2, \ldots}$ is a sequence of independent and identically distributed random variables with distribution function $G(z)(z>0)$ and $E(Z)<\infty$ (i.e. magnitude of contribution of catastrophic event $i$ to intensity);

- $\left\{S_{i}\right\}_{i=1,2, \ldots}$ is the sequence representing the event times of a Poisson process $M_{t}$ with constant intensity $\rho$; and

- $\delta$ is the rate of exponential decay.

Catastrophic events may take long to materialise so the decay rate may not be exponential. It is assumed to be of this form for a matter of convenience, i.e. closed-form expressions of final results are easily derived. We also make the additional assumption that a Poisson process $M_{t}$ and the sequences $\left\{Z_{i}\right\}_{i=1,2, \ldots},\left\{X_{i}\right\}_{i=1,2, \ldots}$ and $\left\{Y_{i}\right\}_{i=1,2, \ldots}$ are independent of each other.

A Poisson process with loss frequency rate $\mu$ is also studied for $N_{t}$, that may be considered when catastrophic loss frequency rate is deterministic.

With the above model specifications, we calculate the following relevant risk measures:

$$
\begin{gathered}
\lim _{u>1} \mathbb{P}\left\{L_{t}^{(1)}>F_{L_{t}^{(1)}}^{-1}(l) \mid L_{t}^{(2)}>F_{L_{t}^{(2)}}^{-1}(l)\right\}, \\
\mathbb{E}\left\{L_{t}^{(1)} \mid L_{t}^{(1)}>F_{L_{t}^{(1)}}^{-1}(l), L_{t}^{(2)}>F_{L_{t}^{(2)}}^{-1}(l)\right\}, \\
\lim _{u>1} \mathbb{P}\left\{L_{t}^{(1)}>F_{L_{t}^{(1)}}^{-1}(l) \mid L_{t}^{(1)}+L_{t}^{(2)}>F_{L_{t}^{(1)}+L_{t}^{(2)}}^{-1}(l)\right\}
\end{gathered}
$$

and

$$
\mathbb{E}\left\{L_{t}^{(1)} \mid L_{t}^{(1)}+L_{t}^{(2)}>F_{L_{t}^{(1)}+L_{t}^{(2)}}^{-1}(l)\right\} .
$$

Here $F_{L_{t}^{(1)}}, F_{L_{t}^{(2)}}$ and $F_{L_{t}^{(1)}+L_{t}^{(2)}}$ are the distribution functions of the random variables $L_{t}^{(1)}, L_{t}^{(2)}$ and $L_{t}^{(1)}+L_{t}^{(2)}$ respectively. The quantities (3) and (5) are known as asymptotic upper tail dependence measures and the quantities (4) and (6) are conditional tail expectations. The motivation for calculating these quantities that measure extremal dependence in the upper tail of a bivariate distribution is that insurance industry is more concerned with dependence between extreme losses. For a discussion on the coefficient of tail dependence parameters, see McNeil et al. [22].

In order to evaluate above risk measures, we need to obtain the joint distribution of the aggregate collateral losses $L_{t}^{(1)}$ and $L_{t}^{(2)}$. Unfortunately, it is not easy to derive the joint distribution the aggregate collateral losses explicitly. So in Section 2, we derive joint Laplace transform of the distribution of the aggregate collateral losses expressed with a copula function applying the piecewise deterministic Markov processes (PDMPs) theory. For $N_{t}$, a shot-noise Cox process and a homogeneous Poisson process are used respectively. Section 3 provides the expressions of the moments, covariance and linear correlation between $L_{t}^{(1)}$ and $L_{t}^{(2)}$ at time $t$ for both cases. In Section 4, we present the expressions for joint probabilities of the aggregate collateral losses and their densities at $L_{t}^{(1)}=0$ and $L_{t}^{(2)}=0$, which are required to improve the accuracy of the distributions of the aggregate collateral losses inverting joint Fast Fourier transforms. We also provide the figures of the joint dis- 
tribution of the aggregate collateral losses and their contours. In Section 5, we illustrate the calculations of relevant risk measures (3)-(6) using joint Fast Fourier transforms. For numerical illustrations, an exponential distribution for the jump sizes of catastrophic event, a member of Farlie-Gumbel-Morgenstern copula with exponential margins are used throughout the paper. Section 6 shows the sensitivity analysis on the parameters of a shot-noise Cox process from a Poisson process using the risk measure of (4). Concluding remarks are in Section 7.

\section{Joint Laplace Transform of the Distribution of the Aggregate Collateral Losses}

The piecewise deterministic Markov processes theory developed by Davis [23] is a powerful mathematical tool for examining non-diffusion models. From now on, we present definitions and important properties of $L_{t}^{(1)}$ and $L_{t}^{(2)}$ with the aid of piecewise deterministic Markov processes theory $[16,24,25]$. This theory is used to derive joint Laplace transform of the distribution of the aggregate collateral losses $L_{t}^{(1)}$ and $L_{t}^{(2)}$.

\subsection{Shot-Noise Cox Process}

Assuming that the loss arrival process $N_{t}$ follows a Cox process with shot noise intensity $\lambda_{t}$, the generator of the process $\left(\lambda_{t}, \Lambda_{t}, N_{t}, L_{t}^{(1)}, L_{t}^{(2)}, t\right)$ acting on a function $f\left(\lambda, \Lambda, n, l^{(1)}, l^{(2)}, t\right)$ belonging to its domain is given by

$$
\begin{aligned}
& A f\left(\lambda, \Lambda, n, l^{(1)}, l^{(2)}, t\right) \\
= & \frac{\partial f}{\partial t}+\lambda \frac{\partial f}{\partial \Lambda} \\
+ & \lambda\left[\int_{0}^{\infty} \int_{0}^{\infty} f\left(\lambda, \Lambda, n+1, l^{(1)}+x, l^{(2)}+y, t\right)\right. \\
& \cdot \frac{\partial^{2} C(F(x), F(y))}{\partial x \partial y} \mathrm{~d} x \mathrm{~d} y \\
& \left.-f\left(\lambda, \Lambda, n, l^{(1)}, l^{(2)}, t\right)\right] \\
- & \delta \lambda \frac{\partial f}{\partial \lambda}+\rho \\
\cdot & {\left[\int_{0}^{\infty} f\left(\lambda+z, \Lambda, n, l^{(1)}, l^{(2)}, t\right) \mathrm{d} G(z)-f\left(\lambda, \Lambda, n, l^{(1)}, l^{(2)}, t\right)\right] }
\end{aligned}
$$

where $\Lambda_{t}=\int_{0}^{t} \lambda_{s} \mathrm{~d} s$. Now let us find a suitable martingale in order to derive joint Laplace transform of the distribution of the aggregate collateral losses $L_{t}^{(1)}$ and
$L_{t}^{(2)}$

Lemma 2.1. Considering constants

$0 \leq \zeta \leq 1, v \geq 0, \xi \geq 0$ and $\kappa \geq 0$ then

$$
\begin{aligned}
& \zeta^{N_{t}} \exp \left(-v L_{t}^{(1)}\right) \exp \left(-\xi L_{t}^{(2)}\right) \\
& \exp \left[-\{\zeta \hat{c}(\nu, \xi)-1\} \Lambda_{t}\right] \\
& \times \exp \left(-\kappa \lambda_{t} \mathrm{e}^{\delta t}\right) \exp \left[\rho \int_{0}^{t}\left\{1-\hat{g}\left(\kappa \mathrm{e}^{\delta s}\right)\right\} \mathrm{d} s\right]
\end{aligned}
$$

is a martingale, where

$$
\begin{aligned}
& \hat{c}(v, \xi)=\int_{0}^{\infty} \int_{0}^{\infty} \mathrm{e}^{-v x} \mathrm{e}^{-\xi y} \frac{\partial^{2} C(F(x), F(y))}{\partial x \partial y} \mathrm{~d} x \mathrm{~d} y<\infty \text { and } \\
& \hat{g}(u)=\int_{0}^{\infty} \mathrm{e}^{-u z} \mathrm{~d} G(z) .
\end{aligned}
$$

Proof. From (7), $f\left(\lambda, \Lambda, n, l^{(1)}, l^{(2)}, t\right)$ has to satisfy $A f=0$ for $f\left(\lambda, \Lambda, n, l^{(1)}, l^{(2)}, t\right)$ to be a martingale. Setting

$$
\begin{aligned}
& f\left(\lambda, \Lambda, n, l^{(1)}, l^{(2)}, t\right) \\
& =\zeta^{n} \mathrm{e}^{\psi \Lambda} \exp \left(-v l^{(1)}\right) \exp \left(-\xi l^{(2)}\right) \exp \left(-\kappa \lambda \mathrm{e}^{\delta t}\right) \mathrm{e}^{B(t)}
\end{aligned}
$$

we get the equation

$$
B^{\prime}(t)+\lambda \psi+\lambda\{\zeta \hat{c}(\nu, \xi)-1\}+\rho\left\{\hat{g}\left(\kappa \mathrm{e}^{\delta t}\right)-1\right\}=0
$$

and the solution is

$$
\psi=-\{\zeta \hat{c}(v, \xi)-1\}, B(t)=\rho \int_{0}^{t}\left\{1-\hat{g}\left(\kappa \mathrm{e}^{\mathrm{d} s}\right)\right\} \mathrm{d} s
$$

by which the result follows.

Using the martingale obtained in Lemma 2.1 and setting $\zeta=1$ and $\kappa=0$, we can easily obtain the general form of joint Laplace transform of the distribution of the aggregate collateral losses $L_{t}^{(1)}$ and $L_{t}^{(2)}$, i.e.

$$
\begin{aligned}
& E\left\{\mathrm{e}^{-v L_{t_{2}}^{(1)}} \mathrm{e}^{-\xi L_{t_{2}}^{(2)}} \mid \mathfrak{J}_{t}\right\} \\
& =\exp \left(-v L_{t_{1}}^{(1)}\right) \exp \left(-\xi L_{t_{1}}^{(2)}\right) \exp \left[\{\hat{c}(v, \xi)-1\} \int_{t_{1}}^{t_{2}} \lambda_{s} \mathrm{~d} s\right]
\end{aligned}
$$

where the conditional expectation $E$ is based on the probability space $(\Omega, \mathfrak{I}, P)$, and the information set $\mathfrak{I}=\left\{\mathfrak{I}_{t}\right\}_{t \geq 0}$ with the filtration

$\mathfrak{J}_{t}=\sigma\left\{\left(\lambda_{s}, L_{s}^{(1)}, L_{s}^{(2)}\right): s \leq t\right\}$. Without loss of generality, change the time scale and assume that $L_{0}^{(1)}=0$ and $L_{0}^{(2)}=0$, then it is given by

$$
E\left\{\mathrm{e}^{-v L_{t}^{(1)}} \mathrm{e}^{-\xi L_{t}^{(2)}} \mid \lambda_{0}\right\}=\exp \left[\{\hat{c}(v, \xi)-1\} \int_{0}^{t} \lambda_{s} \mathrm{~d} s\right] .
$$

Throughout the paper, we firstly assume that jump 
sizes of catastrophic event follow an exponential distribution, i.e. $g(z)=\gamma \exp (-\gamma z), z>0, \gamma>0$ and that $\lambda_{t}$ is stationary. Then using Theorem 2.6 in [16], (12) is given by

$$
\begin{aligned}
& E\left\{\mathrm{e}^{-v L_{t}^{(1)}} \mathrm{e}^{-\xi L_{t}^{(2)}}\right\}=\left(\frac{\gamma \mathrm{e}^{-\delta t}}{\gamma+\frac{1-\hat{c}(v, \xi)}{\delta}\left(1-\mathrm{e}^{-\delta t}\right)}\right)^{\frac{\rho}{\delta}} \\
& \left(\frac{\gamma+\frac{1-\hat{c}(v, \xi)}{\delta}\left(1-\mathrm{e}^{-\delta t}\right)}{\gamma \mathrm{e}^{-\delta t}}\right)^{\frac{\gamma \rho}{\delta \gamma+\{1-\hat{c}(v, \xi)\}}} \cdot
\end{aligned}
$$

Secondly, as a specific example for $C$, we use the Farlie-Gumbel-Morgenstern (FGM) copulas given by

$$
C(u, v)=u v+\theta u v(1-u)(1-v)
$$

where $u \in[0,1], v \in[0,1]$ and $\theta \in[-1,1]$. Lastly, to make this calculation somewhat easier, we assume that $F(x)=1-\mathrm{e}^{-\alpha x}(\alpha>0, x>0)$ and

$F(y)=1-\mathrm{e}^{-\beta y}(\beta>0, y>0)$. We omit the corresponding expression for the above joint Laplace transform of the distribution of the aggregate collateral losses as it can be easily obtained using the joint distribution function $F(x, y)$ driven by (14), i.e.

$$
\begin{aligned}
& \hat{c}(v, \xi) \\
& =1-\frac{(\alpha \xi+\beta v+v \xi)(2 \alpha+v)(2 \beta+\xi)-\theta \alpha \beta v \xi}{(\alpha+v)(\beta+\xi)(2 \alpha+v)(2 \beta+\xi)} .
\end{aligned}
$$

It will be of interest to examine the joint Laplace transform of the distribution of the aggregate collateral losses $L_{t}^{(1)}$ and $L_{t}^{(2)}$ at time $t$, using other copulas and other margins $F(x)$ and $F(y)$. If $\left\{Z_{i}\right\}_{i=1,2, \ldots}$, which are the magnitude of contribution of catastrophic event to intensity $\lambda_{t}$, are high, we also need to consider heavy-tailed distributions for jump size of catastrophic event, $G(z)$. We also omit the corresponding expressions for the Laplace transform of the distribution of $L_{t}^{(i)}, i=1,2$ which are the Laplace transforms of the distribution of the compound Cox process with shot noise intensity $\lambda_{t}$, where its jump sizes follow an exponential distribution [16].

If we set $\theta=0$ in (15), we have joint Laplace transform of the distribution of the aggregate collateral losses, which is the case that two losses $X$ and $Y$ occur same time from a sharing loss frequency rate $\lambda_{t}$, but their sizes are independent each other. Due to the dependence of collateral losses of $X$ and $Y$ with sharing loss frequency rate $\lambda_{t}$, we can see that

$$
E\left\{\mathrm{e}^{-v L_{t}^{(1)}} \mathrm{e}^{-\xi L_{t}^{(2)}}\right\} \neq E\left(\mathrm{e}^{-v L_{t}^{(1)}}\right) E\left(\mathrm{e}^{-\xi L_{t}^{(2)}}\right)
$$

even if $\theta=0$. If loss $X$ occurs by arrival process $N_{t}^{(1)}$ with shot noise intensity $\lambda_{t}^{(1)}$ that has three parameters of $\delta^{(1)}, \quad \rho^{(1)}$ and $G\left(z^{(1)}\right)$ and loss $Y$ occurs by arrival process $N_{t}^{(2)}$ with shot noise intensity $\lambda_{t}^{(2)}$ that has three parameters of $\delta^{(2)}, \rho^{(2)}$ and $G\left(z^{(2)}\right)$ and everything is independent each other, we can have the joint Laplace transform of the distribution of aggregate losses $L_{t}^{(1)}$ and $L_{t}^{(2)}$ at time $t$, that is the product of the Laplace transforms of the distribution of $L_{t}^{(i)}, i=1,2$.

\subsection{Homogeneous Poisson Process}

Let us now assume that the loss arrival process $N_{t}$ follows a homogeneous Poisson process with loss frequency $\mu$. Setting $\lambda_{t}=\mu$ in (12), i.e. considering deterministic loss frequency $\mu$, we can easily obtain that

$$
E\left\{\mathrm{e}^{-v L_{t}^{(1)}} \mathrm{e}^{-\xi L_{t}^{(2)}}\right\}=\exp [-\mu\{1-\hat{c}(v, \xi)\} t]
$$

and using (14) we have

$$
\begin{aligned}
& E\left\{\mathrm{e}^{-v L_{t}^{(1)}} \mathrm{e}^{-\xi L_{t}^{(2)}}\right\}= \\
& \exp \left[-\mu\left\{\frac{(\alpha \xi+\beta v+v \xi)(2 \alpha+v)(2 \beta+\xi)-\theta \alpha \beta v \xi}{(\alpha+v)(\beta+\xi)(2 \alpha+v)(2 \beta+\xi)}\right\} t\right] .
\end{aligned}
$$

We omit the corresponding expressions for the Laplace transform of the distribution of $L_{t}^{(i)}, i=1,2$, which are the Laplace transforms of the distribution of the compound Poisson process with exponential loss sizes, as they can be easily obtained setting $\xi=0$ and $v=0$, respectively.

Similar to shot-noise Cox process for $N_{t}$, due to the dependence of collateral losses of $X$ and $Y$ with sharing loss frequency rate $\mu$, it shows that

$$
E\left\{\mathrm{e}^{-\nu L_{t}^{(1)}} \mathrm{e}^{-\xi L_{t}^{(2)}}\right\} \neq E\left(\mathrm{e}^{-\nu L_{t}^{(1)}}\right) E\left(\mathrm{e}^{-\xi L_{t}^{(2)}}\right)
$$

even if $\theta=0$. If loss $X$ occurs by arrival process $N_{t}^{(1)}$ with loss frequency $\mu^{(1)}$ and loss $Y$ occurs by arrival process $N_{t}^{(2)}$ with loss frequency $\mu^{(2)}$ and everything is independent each other, we can also have the joint Laplace transform of the distribution of aggregate losses $L_{t}^{(1)}$ and $L_{t}^{(2)}$ at time $t$ that is the product of the Laplace transforms of the distribution of $L_{t}^{(i)}, i=1,2$.

\section{Moments, Covariance and Linear Correlation of Aggregate Collateral Losses}

In this section, we examine the moments, covariance and 
linear correlation between $L_{t}^{(1)}$ and $L_{t}^{(2)}$ at time $t$. For the loss arrival process $N_{t}$, we use a Cox process with shot noise intensity $\lambda_{t}$ and a homogeneous Poisson process with loss frequency $\mu$, respectively.

\subsection{Shot-Noise Cox Process}

Differentiating (12) w.r.t. $v$ and $\xi$ and set $v=0$ and $\xi=0$, we can derive the joint expectation of $L_{t}^{(1)}$ and $L_{t}^{(2)}$ at time $t$, i.e.

$$
E\left\{L_{t}^{(1)} L_{t}^{(2)}\right\}=E\left(\Lambda_{t}\right) E(X Y)+E\left(\Lambda_{t}^{2}\right) E(X) E(Y) .
$$

Also set $\xi=0$ and $v=0$ in (12) and differentiate itw.r.t. $v$ and $\xi$ respectively, we can obtain the expectation of $L_{t}^{(1)}$ and $L_{t}^{(2)}$ at time $t$, i.e.

$$
E\left\{L_{t}^{(1)}\right\}=E\left(\Lambda_{t}\right) E(X)
$$

and

$$
E\left\{L_{t}^{(2)}\right\}=E\left(\Lambda_{t}\right) E(Y) .
$$

The higher moments of $L_{t}^{(1)}$ and $L_{t}^{(2)}$ at time $t$ can be obtained by differentiating it further, i.e.

$$
\operatorname{Var}\left\{L_{t}^{(1)}\right\}=E\left(\Lambda_{t}\right) E\left(X^{2}\right)+\operatorname{Var}\left(\Lambda_{t}\right)\{E(X)\}^{2}
$$

and

$$
\operatorname{Var}\left\{L_{t}^{(2)}\right\}=E\left(\Lambda_{t}\right) E\left(Y^{2}\right)+\operatorname{Var}\left(\Lambda_{t}\right)\{E(Y)\}^{2} .
$$

The covariance between $L_{t}^{(1)}$ and $L_{t}^{(2)}$ at time $t$ is given by

$$
\operatorname{Cov}\left(L_{t}^{(1)}, L_{t}^{(2)}\right)=E\left(\Lambda_{t}\right) E(X Y)+\operatorname{Var}\left(\Lambda_{t}\right) E(X) E(Y)
$$

and the linear correlation coefficient between $L_{t}^{(1)}$ and $L_{t}^{(2)}$ at time $t$ is given by

$$
\begin{aligned}
& \rho\left(L_{t}^{(1)}, L_{t}^{(2)}\right) \\
& =\frac{E\left(\Lambda_{t}\right) E(X Y)+\operatorname{Var}\left(\Lambda_{t}\right) E(X) E(Y)}{\sqrt{\left\{E\left(\Lambda_{t}\right)\right\}^{2} E\left(X^{2}\right) E\left(Y^{2}\right)+E\left(\Lambda_{t}\right) \operatorname{Var}\left(\Lambda_{t}\right)\left[E\left(X^{2}\right)\{E(Y)\}^{2}+\{E(X)\}^{2} E\left(Y^{2}\right)\right]+\left\{\operatorname{Var}\left(\Lambda_{t}\right) E(X) E(Y)\right\}^{2}}}
\end{aligned}
$$

Let us now illustrate the calculations of the covariance and linear correlation between $L_{t}^{(1)}$ and $L_{t}^{(2)}$ at time $t$, where $N_{t}$ follows a shot-noise Cox process.

\section{Example 1}

From [17], we have

$$
E\left(\Lambda_{t}\right)=\frac{\rho}{\gamma \delta} t, \operatorname{Var}\left(\Lambda_{t}\right)=\frac{2 \rho}{\gamma^{2} \delta^{2}} t+\frac{2 \rho}{\gamma^{2} \delta^{3}} \mathrm{e}^{-\delta t}-\frac{2 \rho}{\gamma^{2} \delta^{3}}
$$

and using (14) we have

$$
E(X Y)=\frac{1}{\alpha \beta}\left(1+\frac{\theta}{4}\right) .
$$

The parameter values used to calculate the covariance and linear correlation using (25) and (26) are

$$
\delta=0.5, \rho=2, \gamma=1, \alpha=1, \beta=0.5, t=1 .
$$

Hence from (25) and (26), the calculations of covariance and linear correlation between $L_{t}^{(1)}$ and $L_{t}^{(2)}$ at time $t$ are shown in Tables $\mathbf{1}$ and $\mathbf{2}$ respectively.

\subsection{Homogeneous Poisson Process}

Similar to a shot-noise Cox process for $N_{t}$, differentiating (17) w.r.t. $v$ and $\xi$ and set $v=0$ and $\xi=0$ we can easily derive the joint expectation of $L_{t}^{(1)}$, and $L_{t}^{(2)}$ at time $t$, i.e.

$$
E\left\{L_{t}^{(1)} L_{t}^{(2)}\right\}=\mu t E(X Y)+(\mu t)^{2} E(X) E(Y) .
$$

We omit the expressions for the expectation and variance of $L_{t}^{(1)}$ and $L_{t}^{(2)}$ at time $t$ as they can be easily derived similar to a shot-noise Cox process for $N_{t}$.

The covariance between $L_{t}^{(1)}$ and $L_{t}^{(2)}$ at time $t$ is given by

$$
\operatorname{Cov}\left(L_{t}^{(1)}, L_{t}^{(2)}\right)=\mu t E(X Y)
$$

and the linear correlation coefficient between $L_{t}^{(1)}$ and $L_{t}^{(2)}$ at time $t$ is given by

$$
\rho\left(L_{t}^{(1)}, L_{t}^{(2)}\right)=\frac{E(X Y)}{\sqrt{E\left(X^{2}\right) E\left(Y^{2}\right)}} .
$$

Let us now illustrate the calculations of the covariance and linear correlation between $L_{t}^{(1)}$ and $L_{t}^{(2)}$ at time $t$, where $N_{t}$ follows a Poisson process.

\section{Example 2}

The parameter values used to calculate the covariance and linear correlation using (28) and (29) are

$$
\mu=4, \alpha=1, \beta=0.5, t=1 \text {. }
$$

From (28) and (29), the calculations of covariance and linear correlation between $L_{t}^{(1)}$ and $L_{t}^{(2)}$ at time $t$ are shown in Table 3 and Table 4 respectively. 
Table 1. Covariance between $L_{t}^{(1)}$ and $L_{t}^{(2)}$.

\begin{tabular}{cc}
\hline$\theta$ & $\operatorname{Cov}\left(L_{t}^{(1)}, L_{t}^{(2)}\right)$ \\
\hline-1 & 12.82 \\
-0.5 & 13.82 \\
0 & 14.82 \\
0.5 & 15.82 \\
1 & 16.82 \\
\hline
\end{tabular}

Table 2. Linear correlation between $L_{t}^{(1)}$ and $L_{t}^{(2)}$.

\begin{tabular}{cc}
\hline$\theta$ & $\rho\left(L_{t}^{(1)}, L_{t}^{(2)}\right)$ \\
\hline-1 & 0.56175 \\
-0.5 & 0.60557 \\
0 & 0.64940 \\
0.5 & 0.69322 \\
1 & 0.73705 \\
\hline
\end{tabular}

Table 3. Covariance between $L_{t}^{(1)}$ and $L_{t}^{(2)}$.

\begin{tabular}{cc}
\hline$\theta$ & $\operatorname{Cov}\left(L_{t}^{(1)}, L_{t}^{(2)}\right)$ \\
\hline-1 & 6 \\
-0.5 & 7 \\
0 & 8 \\
0.5 & 9 \\
1 & 10 \\
\hline
\end{tabular}

Table 4. Linear correlation between $L_{t}^{(1)}$ and $L_{t}^{(2)}$.

\begin{tabular}{cc}
\hline$\theta$ & $\rho\left(L_{t}^{(1)}, L_{t}^{(2)}\right)$ \\
\hline-1 & 0.375 \\
-0.5 & 0.4375 \\
0 & 0.5 \\
0.5 & 0.5625 \\
1 & 0.625
\end{tabular}

\subsection{Comparison}

The parameter values used in Examples 1-2 provide us with the same means of aggregate collateral losses regardless of the loss arrival process $N_{t}$, i.e.

$$
\mathbb{E}^{\text {Poisson }}\left\{L_{t}^{(k)}\right\}=\mathbb{E}^{\text {Cox }}\left\{L_{t}^{(k)}\right\} \text {, }
$$

where

$$
\mathbb{E}^{\text {Poisson }}\left\{L_{t}^{(1)}\right\}=\mathbb{E}^{\text {Cox }}\left\{L_{t}^{(1)}\right\}=4
$$

and

$$
\mathbb{E}^{\text {Poisson }}\left\{L_{t}^{(2)}\right\}=\mathbb{E}^{\text {Cox }}\left\{L_{t}^{(2)}\right\}=8 .
$$

However for each $\theta$, Tables 1 and $\mathbf{3}$ show that there is an increase in the covariance between $L_{t}^{(1)}$ and $L_{t}^{(2)}$ by changing $N_{t}$ from a homogenous Poisson process to a shot-noise Cox process. Tables $\mathbf{2}$ and $\mathbf{4}$ also show that the linear correlation between $L_{t}^{(1)}$ and $L_{t}^{(2)}$ increases by changing $N_{t}$ from a homogenous Poisson process to a shot-noise Cox process for each $\theta$. This implies that the marginal distributions of the aggregate collateral loss with respect to a Cox process have heavier tail than their counterparts with respect to a Poisson process, i.e.

$$
\operatorname{Var}^{\text {Poisson }}\left\{L_{t}^{(1)}\right\}=8<\operatorname{Var}^{\operatorname{Cox}}\left\{L_{t}^{(1)}\right\}=11.41
$$

and

$$
\operatorname{Var}^{\text {Poisson }}\left\{L_{t}^{(2)}\right\}=32<\operatorname{Var}^{\operatorname{Cox}}\left\{L_{t}^{(2)}\right\}=45.64
$$

It will also become apparent by the joint distributions of aggregate collateral losses and their contours in Section 4 and numerical risk measure values in Examples 36.

\section{Joint Distribution of the Aggregate Collateral Losses via Bivariate Fast Fourier Transform}

In order to calculate the risk measures of (3)-(6), we invert bivariate Fast Fourier transforms from joint Laplace transforms of the vector $\left(L_{t}^{(1)}, L_{t}^{(2)}\right)$ obtained in Section 2. For details on how to use bivariate Fast Fourier transform, we refer to [26-28]. Before we show the calculations of risk measures in Section 5, we present the expressions for the joint probabilities of the aggregate collateral losses and their densities at $L_{t}^{(1)}=0$ and $L_{t}^{(2)}=0$. These are required to improve the accuracy of the joint distributions of the aggregate collateral losses inverting bivariate Fast Fourier transforms.

\subsection{Shot-Noise Cox Process}

If we let $v \rightarrow \infty$ and $\xi \rightarrow \infty$ in (13), we have the expression for the joint probability of aggregate collateral losses at $L_{t}^{(1)}=0$ and $L_{t}^{(2)}=0$, i.e.

$$
\mathbb{P}\left(L_{t}^{(1)}=0, L_{t}^{(2)}=0\right)=\left(\frac{\delta \gamma \mathrm{e}^{-\delta t}}{1-\mathrm{e}^{-\delta t}+\delta \gamma}\right)^{\frac{\rho}{\delta(1+\delta \gamma)}} .
$$

Regardless of loss size distributions, we have the same joint probability of aggregate collateral losses at $L_{t}^{(1)}=0$ and $L_{t}^{(2)}=0$ when the loss arrival process $N_{t}$ follows a Cox process with shot noise intensity $\lambda_{t}$. If we set 


$$
\lim _{v \rightarrow \infty, \xi \rightarrow \infty} v \xi\left[\left(\frac{\gamma \mathrm{e}^{-\delta t}}{\gamma+\frac{1-\hat{c}(v, \xi)}{\delta}\left(1-\mathrm{e}^{-\delta t}\right)}\right)^{\frac{\rho}{\delta}}\left(\frac{\gamma+\frac{1-\hat{c}(v, \xi)}{\delta}\left(1-\mathrm{e}^{-\delta t}\right)}{\gamma \mathrm{e}^{-\delta t}}\right)^{\frac{\gamma \rho}{\delta \gamma+\{1-\hat{c}(\nu, \xi)\}}}-\left(\frac{\delta \gamma \mathrm{e}^{-\delta t}}{1-\mathrm{e}^{-\delta t}+\delta \gamma}\right)^{\frac{\rho}{\delta(1+\delta \gamma)}}\right],
$$

the expression for the joint density of aggregate collateral losses at $L_{t}^{(1)}=0$ and $L_{t}^{(2)}=0$ is given by

$$
\frac{f_{X, Y}(0,0) \times \rho}{1+\delta \gamma}\left(\frac{\delta \gamma \mathrm{e}^{-\delta t}}{1-\mathrm{e}^{-\delta t}+\delta \gamma}\right)^{\frac{\rho}{\delta(1+\delta \gamma)}} \times\left\{\frac{\gamma}{1+\delta \gamma} \ln \left(\frac{1-\mathrm{e}^{-\delta t}+\delta \gamma}{\delta \gamma \mathrm{e}^{-\delta t}}\right)+\frac{1-\mathrm{e}^{-\delta t}}{\delta\left(1-\mathrm{e}^{-\delta t}+\delta \gamma\right)}\right\} .
$$

Based on (35), we can easily obtain its expression for exponential loss sizes using (14), i.e.

$$
\frac{(1+\theta) \alpha \beta \rho}{1+\delta \gamma}\left(\frac{\delta \gamma \mathrm{e}^{-\delta t}}{1-\mathrm{e}^{-\delta t}+\delta \gamma}\right)^{\frac{\rho}{\delta(1+\delta \gamma)}} \times\left\{\frac{\gamma}{1+\delta \gamma} \ln \left(\frac{1-\mathrm{e}^{-\delta t}+\delta \gamma}{\delta \gamma \mathrm{e}^{-\delta t}}\right)+\frac{1-\mathrm{e}^{-\delta t}}{\delta\left(1-\mathrm{e}^{-\delta t}+\delta \gamma\right)}\right\} .
$$

Figures 1-4 are the joint distributions of aggregate collateral losses and their contours at each value of $\theta$ with respect to a shot-noise Cox process for $N_{t}$.

Figure 2 (or Figure 1) shows that joint probabilities of aggregate collateral losses are mainly located between the bottom left corner and the top right corner when $\theta=1$, which means loss $X$ and $Y$ move in the same direction. On the other hand, compared to when $\theta=1$, Figure 4 (or Figure 3) shows that joint probabilities of aggregate collateral losses at the bottom left corner and the top right corner moves to its diagonal left and right, respectively when $\theta=-1$ which means loss $X$ and $Y$ move in the opposite direction.

\subsection{Homogeneous Poisson Process}

If we let $v \rightarrow \infty$ and $\xi \rightarrow \infty$ in (18), we have the expression for the joint probability of aggregate collateral losses at $L_{t}^{(1)}=0$ and $L_{t}^{(2)}=0$, i.e.

$$
\mathbb{P}\left(L_{t}^{(1)}=0, L_{t}^{(2)}=0\right)=\mathrm{e}^{-\mu t} .
$$

Regardless of loss size distributions, we have the same joint probability of aggregate collateral losses at $L_{t}^{(1)}=0$ and $L_{t}^{(2)}=0$ when the loss arrival process $N_{t}$ follows a homogeneous Poisson process with loss frequency $\mu$. If we set

$$
\lim _{v \rightarrow \infty, \xi \rightarrow \infty} v \xi\left[\exp \left[-\mu\left\{\frac{(\alpha \xi+\beta v+v \xi)(2 \alpha+v)(2 \beta+\xi)-\theta \alpha \beta v \xi}{(\alpha+v)(\beta+\xi)(2 \alpha+v)(2 \beta+\xi)}\right\} t\right]-\mathrm{e}^{-\mu t}\right],
$$

the expression for the joint density of aggregate collateral losses at $L_{t}^{(1)}=0$ and $L_{t}^{(2)}=0$ is given by

$$
f_{X, Y}(0,0) \times \mu t \mathrm{e}^{-\mu t} .
$$

Based on (38), we can easily obtain its expression for exponential loss sizes using (14), i.e.

$$
(1+\theta) \alpha \beta \times \mu t \mathrm{e}^{-\mu t} .
$$

The figures of the joint distributions of aggregate collateral losses and their contours at each value of $\theta$ with respect to a Poisson process for $N_{t}$ are omitted, for which see the early version of this paper in http://ssrn.com/author=383758.

\section{Calculating Risk Measures for Collateral Losses}

Now using the parameter values in Examples 1-2 and Matlab, let us illustrate the calculations of the risk measures of (3)-(6) with respect to a shot-noise Cox process and a Poisson process. They are shown in Tables 5-18.

To reduce the error of used algorithm for inverting bivariate Fast Fourier transforms using Matlab, the following methods have been used:

- The sampling points have been taken as many as possible, i.e. we have used $8192 \times 4096$ points in the calculation, which was the maximum points we could sampled in 32-bit Matlab.

- If the sampling interval gap is too small, there would be a large amount of truncation error. Also if the sampling interval is too big, there would be a large amount of sampling error. Hence we have chosen carefully the right sampling interval, so that the total errors caused by inverting bivariate Fast Fourier transforms could be negligible.

- For the figures in this paper, we have used the 2D 


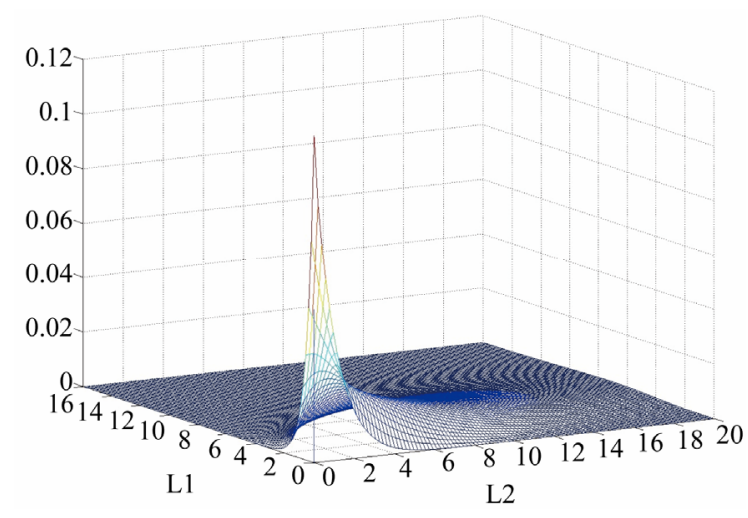

Figure 1. The joint distribution of collateral losses with $\theta=$ 1.

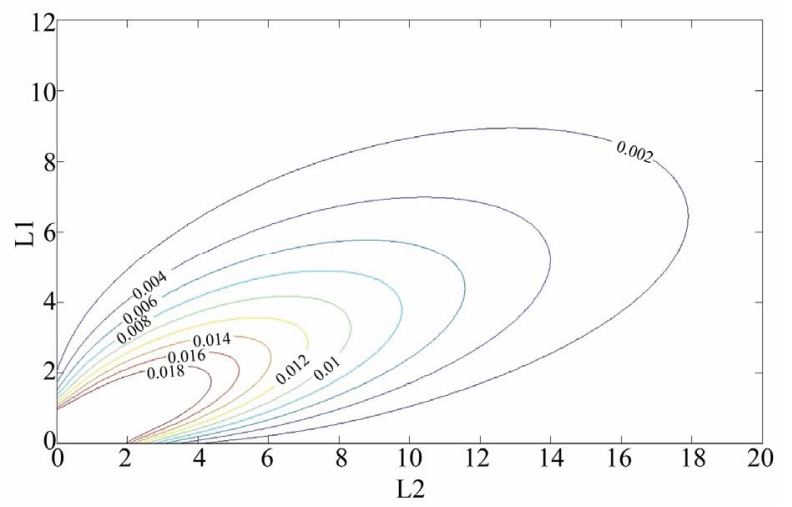

Figure 2. The contour of the joint distribution of collateral losses with $\theta=1$.

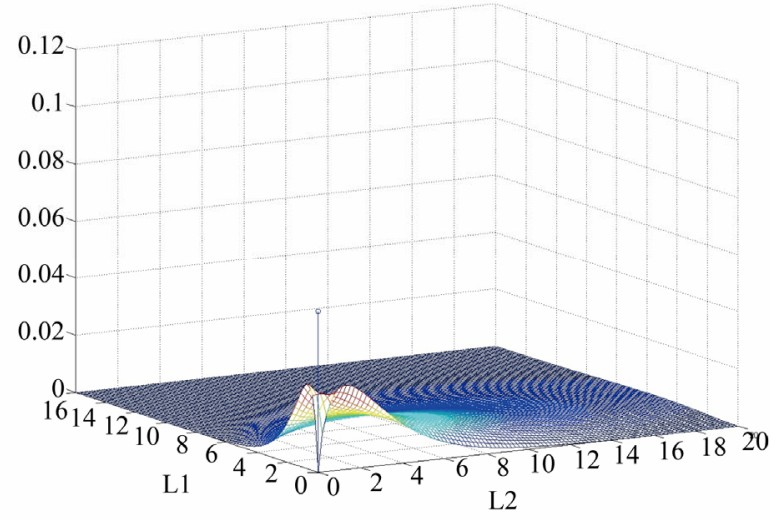

Figure 3. The joint distribution of collateral losses with $\theta=$ -1 .

low-pass/moving-average filter to filter the jitter noise out. Then we have used the linear interpolation to smooth the borders of the data. The pattern matrix used for this filter was

$$
\left[\begin{array}{ccc}
0.0625 & 0.125 & 0.0625 \\
0.125 & 0.25 & 0.125 \\
0.0625 & 0.125 & 0.0625
\end{array}\right] .
$$

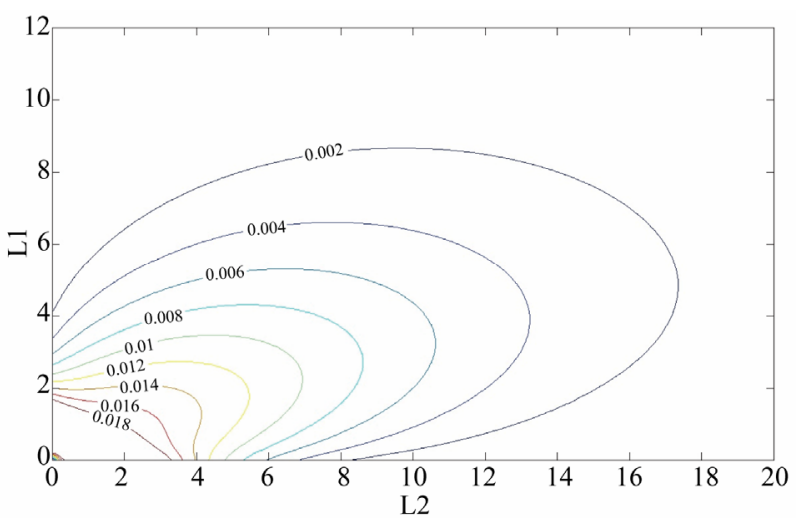

Figure 4. The contour of the joint distribution of collateral losses with $\theta=-1$.

\section{Example 3}

Using the different $\operatorname{VaR}_{q \%}$ at $q \%=95 \%$ and $99 \%$, the calculations of the risk measure of (3) are shown in Tables 5-8.

Tables 5-8 show that each quantile values are higher with respect to a shot-noise Cox process than their counterparts. They also show that the risk measures of (3) with respect to a shot-noise Cox process are higher than their counterparts. These justify that the marginal/joint distributions of the aggregate collateral losses with respect to a shot-noise Cox process have heavier tail than their counterparts with respect to a Poisson process.

Example 4

Secondly, based on the $V a R_{q \%}$ at $95 \%$ and $99 \%$ in Example 3, the calculations of the risk measure (4), i.e. $\mathbb{E}\left\{L_{t}^{(1)} \mid L_{t}^{(1)}>F_{L_{t}^{(1)}}^{-1}(u), L_{t}^{(2)}>F_{L_{t}^{(2)}}^{-1}(u)\right\}$ are shown in Tables 9 and 10.

Tables 9 and $\mathbf{1 0}$ show that the risk measures of (4) with respect to a shot-noise Cox process are higher than their counterparts regardless of the critical values. Regardless of the loss arrival process $N_{t}$, the risk measures of (4) are getting bigger as the critical value goes to $99 \%$. They also show that the differences between the values in Table 9 and their counterparts in Table $\mathbf{1 0}$ are getting higher as the critical value goes to $99 \%$.

\section{Example 5}

Showing the calculations of the $q \%$ quantiles of the sum, $L_{t}^{(1)}+L_{t}^{(2)}$, i.e. $F_{L_{t}^{(1)}+L_{t}^{(2)}}^{-1}(u)$ in $\mathbb{P}\left\{L_{t}^{(1)}+L_{t}^{(2)}>F_{L_{t}^{(1)}+L_{t}^{(2)}}^{-1}(u)\right\}$ and the joint probability between $L_{t}^{(1)}>F_{L_{t}^{(1)}}^{-1}(u)$ and $L_{t}^{(1)}+L_{t}^{(2)}>F_{L_{t}^{(1)}+L_{t}^{(2)}}^{-1}(u)$, i.e. $\mathbb{P}\left\{L_{t}^{(1)}>F_{L_{t}^{(1)}}^{-1}(u), L_{t}^{(1)}+L_{t}^{(2)}>F_{L_{t}^{(1)}+L_{t}^{(2)}}^{-1}(u)\right\}$ for each cases in Tables 11-14, the calculations of the risk measure (5) are shown in Tables $\mathbf{1 5}$ and $\mathbf{1 6 .}$ 
Table 5. A shot-noise Cox process, where $\mathbb{P}\left\{L_{t}^{(1)}>10.55\right\}=\mathbb{P}\left\{L_{t}^{(2)}>21.10\right\}=0.05$.

\begin{tabular}{ccc}
\hline$\theta$ & $\mathbb{P}\left\{L_{t}^{(1)}>10.55, L_{t}^{(2)}>21.10\right\}$ & $\mathbb{P}\left\{L_{t}^{(1)}>10.55 \mid L_{t}^{(2)}>21.10\right\}=\mathbb{P}\left\{L_{t}^{(2)}>21.10 \mid L_{t}^{(1)}>10.55\right\}$ \\
\hline 1 & 0.02090 & 0.41793 \\
0.5 & 0.01900 & 0.38002 \\
0 & 0.01723 & 0.34449 \\
-1 & 0.01555 & 0.31105 \\
\hline
\end{tabular}

Table 6. A Poisson process, where $\mathbb{P}\left\{L_{t}^{(1)}>9.37\right\}=\mathbb{P}\left\{L_{t}^{(2)}>18.74\right\}=0.05$.

\begin{tabular}{ccc}
\hline$\theta$ & $\mathbb{P}\left\{L_{t}^{(1)}>9.37, L_{t}^{(2)}>18.74\right\}$ & $\mathbb{P}\left\{L_{t}^{(1)}>9.37 \mid L_{t}^{(2)}>18.74\right\}=\mathbb{P}\left\{L_{t}^{(2)}>18.74 \mid L_{t}^{(1)}>9.37\right\}$ \\
\hline 1 & 0.01459 & 0.29186 \\
0.5 & 0.01257 & 0.25129 \\
0 & 0.01068 & 0.21363 \\
-0.5 & 0.00893 & 0.17862 \\
-1 & 0.00730 & 0.14605 \\
\hline
\end{tabular}

Table 7. A shot-noise Cox process, where $\mathbb{P}\left\{L_{t}^{(1)}>14.79\right\}=\mathbb{P}\left\{L_{t}^{(2)}>\mathbf{2 9 . 5 8}\right\}=0.01$.

\begin{tabular}{ccc}
\hline$\theta$ & $\mathbb{P}\left\{L_{t}^{(1)}>14.79, L_{t}^{(2)}>29.58\right\}$ & $\mathbb{P}\left\{L_{t}^{(1)}>14.79 \mid L_{t}^{(2)}>29.58\right\}=\mathbb{P}\left\{L_{t}^{(2)}>29.58 \mid L_{t}^{(1)}>14.79\right\}$ \\
\hline 1 & 0.00284 & 0.28389 \\
0.5 & 0.00247 & 0.24727 \\
0 & 0.00214 & 0.21401 \\
-0.5 & 0.00184 & 0.18373 \\
-1 & 0.00156 & 0.15617 \\
\hline
\end{tabular}

Table 8. A Poisson process, where $\mathbb{P}\left\{L_{t}^{(1)}>12.61\right\}=\mathbb{P}\left\{L_{t}^{(2)}>25.22\right\}=0.01$.

\begin{tabular}{ccc}
\hline$\theta$ & $\mathbb{P}\left\{L_{t}^{(1)}>12.61, L_{t}^{(2)}>25.22\right\}$ & $\mathbb{P}\left\{L_{t}^{(1)}>12.61 \mid L_{t}^{(2)}>25.22\right\}=\mathbb{P}\left\{L_{t}^{(2)}>25.22 \mid L_{t}^{(1)}>12.61\right\}$ \\
\hline 1 & 0.00152 & 0.15183 \\
0.5 & 0.00121 & 0.12079 \\
0 & 0.00094 & 0.09369 \\
-0.5 & 0.00070 & 0.07022 \\
-1 & 0.00050 & 0.05013 \\
\hline
\end{tabular}

Table 9. A shot-noise Cox process.

\begin{tabular}{cccc}
\hline$\theta \quad \% \%$ & $95 \%$ & $99 \%$ \\
\hline 1 & 13.99969 & 18.18178 \\
0.5 & 13.99910 & 18.17480 \\
0 & 13.98970 & 18.15887 \\
-0.5 & 13.97155 & 18.13402 \\
-1 & 13.94446 & 18.09996 \\
\hline
\end{tabular}

Table 10. A Poisson process.

\begin{tabular}{cccc}
\hline$\theta \quad q \%$ & $95 \%$ & $99 \%$ \\
\hline 1 & 11.89360 & 14.99842 \\
0.5 & 11.85723 & 14.95484 \\
0 & 11.80964 & 14.90020 \\
-0.5 & 11.74893 & 14.83189 \\
-1 & 11.67172 & 14.74505 \\
\hline
\end{tabular}


Table 11. $q \%$ quantiles of the sum, $L_{t}^{(1)}+L_{t}^{(2)}$ for a shotnoise Cox process.

\begin{tabular}{|c|c|c|}
\hline$\theta \quad q \%$ & $95 \%$ & $99 \%$ \\
\hline 1 & 30.27695 & 41.70510 \\
\hline 0.5 & 30.04685 & 41.32161 \\
\hline 0 & 29.81675 & 40.93811 \\
\hline-0.5 & 29.58665 & 40.59297 \\
\hline-1 & 29.35656 & 40.17112 \\
\hline
\end{tabular}

Table 12. $q \%$ quantiles of the sum, $L_{t}^{(1)}+L_{t}^{(2)}$ for a poisson process

\begin{tabular}{cccc}
\hline$\theta$ & $q \%$ & $95 \%$ & $99 \%$ \\
\hline 1 & 26.44199 & 34.68714 \\
0.5 & 26.17355 & 34.22695 \\
0 & 25.90510 & 33.80510 \\
-0.5 & 25.63665 & 33.30656 \\
-1 & 25.32986 & 32.80801 \\
\hline
\end{tabular}

Table 13. $\mathbb{P}\left\{L_{t}^{(1)}>F_{L_{t}^{(1)}}^{-1}(u), L_{t}^{(1)}+L_{t}^{(2)}>F_{L_{t}^{(1)}+L_{t}^{(2)}}^{-1}(u)\right\} \quad$ for a shot-noise Cox process.

\begin{tabular}{cccc}
\hline$\theta$ & $q \%$ & $95 \%$ & $99 \%$ \\
\hline 1 & 0.02942 & 0.00469 \\
0.5 & 0.02798 & 0.00439 \\
0 & 0.02657 & 0.00410 \\
-0.5 & 0.02519 & 0.00380 \\
-1 & 0.02384 & 0.00354
\end{tabular}

Table 14. $\mathbb{P}\left\{L_{t}^{(1)}>F_{L_{t}^{(1)}}^{-1}(u), L_{t}^{(1)}+L_{t}^{(2)}>F_{L_{t}^{(1)}+L_{t}^{(2)}}^{-1}(u)\right\} \quad$ for a Poisson process.

\begin{tabular}{cccc}
\hline$\theta$ & $q \%$ & $95 \%$ & $99 \%$ \\
\hline 1 & 0.02444 & 0.00344 \\
0.5 & 0.02264 & 0.00311 \\
0 & 0.02085 & 0.00275 \\
-0.5 & 0.01906 & 0.00243 \\
-1 & 0.01736 & 0.00210 \\
\hline
\end{tabular}

Table 15. $\mathbb{P}\left\{L_{t}^{(1)}>F_{L_{t}^{(1)}}^{-1}(u) \mid L_{t}^{(1)}+L_{t}^{(2)}>F_{L_{t}^{(1)}+L_{t}^{(2)}}^{-1}(u)\right\} \quad$ for a shot-noise Cox process.

\begin{tabular}{cccc}
\hline$\theta$ & $q \%$ & $95 \%$ & $99 \%$ \\
\hline 1 & 0.58843 & 0.46900 \\
0.5 & 0.55950 & 0.43908 \\
0 & 0.53137 & 0.40990 \\
-0.5 & 0.50387 & 0.38020 \\
-1 & 0.47681 & 0.35389
\end{tabular}

Table 16. $\mathbb{P}\left\{L_{t}^{(1)}>F_{L_{t}^{(1)}}^{-1}(u) \mid L_{t}^{(1)}+L_{t}^{(2)}>F_{L_{t}^{(1)}+L_{t}^{(2)}}^{-1}(u)\right\}$ for a Poisson process.

\begin{tabular}{ccc}
\hline$\theta q \%$ & $95 \%$ & $99 \%$ \\
\hline 1 & 0.48882 & 0.34377 \\
0.5 & 0.45288 & 0.31048 \\
0 & 0.41706 & 0.27504 \\
-0.5 & 0.38126 & 0.24282 \\
-1 & 0.34710 & 0.21026 \\
\hline
\end{tabular}

Table 17. A shot-noise Cox process.

\begin{tabular}{cccc}
\hline$\theta$ & $q \%$ & $95 \%$ & $99 \%$ \\
\hline 1 & 11.69317 & 14.99356 \\
0.5 & 11.46820 & 14.64166 \\
0 & 11.22903 & 14.25449 \\
-0.5 & 10.97467 & 13.75803 \\
-1 & 10.70397 & 13.36212 \\
\hline
\end{tabular}

Table 18. A Poisson process.

\begin{tabular}{cccc}
\hline$\theta$ & $q \%$ & $95 \%$ & $99 \%$ \\
\hline 1 & 9.57781 & 11.53855 \\
0.5 & 9.28678 & 11.14740 \\
0 & 8.96709 & 10.60372 \\
-0.5 & 8.61560 & 10.13953 \\
-1 & 8.28519 & 9.57822 \\
\hline
\end{tabular}


Tables 11 and 12 show that the $q \%$ quantiles of the sum, $L_{t}^{(1)}+L_{t}^{(2)}$ with respect to a shot-noise Cox process are higher than their counterparts regardless of the critical values. Regardless of the loss arrival process $N_{t}$, they are getting bigger as the critical value goes to $99.9 \%$. They also show that the differences between the values in Table 11 and their counterparts in Table 12 are getting higher as the critical value goes to $99.9 \%$.

Tables 13 and 14 show that the joint probability between $L_{t}^{(1)}>F_{L_{t}^{(1)}}^{-1}(u)$ and $L_{t}^{(1)}+L_{t}^{(2)}>F_{L_{t}^{(1)}+L_{t}^{(2)}}^{-1}(u)$ with respect to a shot-noise Cox process are higher than their counterparts regardless of the critical values. Regardless of the loss arrival process $N_{t}$, they are getting smaller as the critical value goes to $99.9 \%$. They also show that the differences between the values in Table 13 and their counterparts in Table $\mathbf{1 4}$ are getting lower as the critical value goes to $99.9 \%$.

Tables 15 and 16 show that the risk measures of (5) with respect to a shot-noise Cox process are higher than their counterparts regardless of the critical values. Regardless of the loss arrival process $N_{t}$, the risk measures of (5) are getting smaller as the critical value goes to $99.9 \%$.

\section{Example 6}

Lastly, based on the quantile values and the joint probabilities in Example 5, the calculations of the risk measure (6), i.e. $\mathbb{E}\left\{L_{t}^{(1)} \mid L_{t}^{(1)}+L_{t}^{(2)}>F_{L_{t}^{(1)}+L_{t}^{(2)}}^{-1}(u)\right\}$ are shown in Tables 17 and 18.

Tables 17 and 18 show that the risk measures of (6) with respect to a shot-noise Cox process are higher than their counterparts regardless of the quantile values. Regardless of the loss arrival process $N_{t}$, the risk measures of (6) are getting bigger as the critical value goes to $99.9 \%$. They also show that the differences between the values in Table 17 and their counterparts in Table 18 are getting higher as the critical value goes to $99.9 \%$.

With respect to the FGM copula correlation parameter, $\theta$, the risk measures of (3)-(6) are increasing (decreasing) as it changes to $1(-1)$ regardless of the loss arrival process $N_{t}$. It will be interesting to find these risk measure values using other copulas with different marginal distributions.

\section{Sensitivity Analysis}

In this section, we examine the effect on the risk measure of (4), i.e.

$$
\mathbb{E}\left\{L_{t}^{(1)} \mid L_{t}^{(1)}>F_{L_{t}^{(1)}}^{-1}(u), L_{t}^{(2)}>F_{L_{t}^{(2)}}^{-1}(u)\right\}
$$

caused by changes in the values of the parameters of a shot-noise Cox process from a Poisson process. To do so, we double the means of aggregate collateral losses, (30) and (31) with $\mu=8$ for a Poisson case and with $\rho=4, \delta=0.25$ and $\gamma=0.5$, respectively for shotnoise Cox case, i.e.

$$
\mathbb{E}^{\text {Poisson }}\left\{L_{t}^{(1)}\right\}=\mathbb{E}^{\text {Cox }}\left\{L_{t}^{(1)}\right\}=8
$$

and

$$
\mathbb{E}^{\text {Poisson }}\left\{L_{t}^{(2)}\right\}=\mathbb{E}^{\text {Cox }}\left\{L_{t}^{(2)}\right\}=16,
$$

where other parameter values are the same as in Examples 1-2.

Figures 5-6 are the joint distribution of aggregate collateral losses and its contour at $\theta=1$ with respect to a Poisson process for $N_{t}$. Figures 7-12 are the joint distributions of aggregate collateral losses and their con-

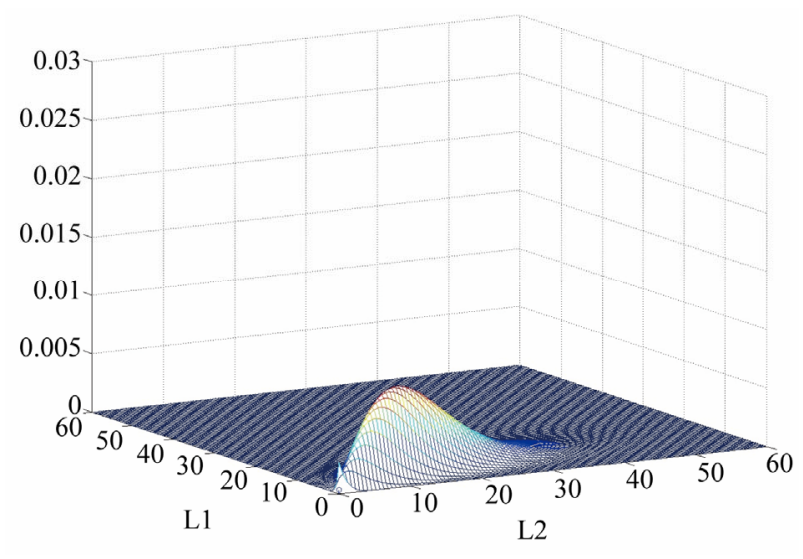

Figure 5. The joint distribution of collateral losses with $\theta=$ 1 for a Poisson process.

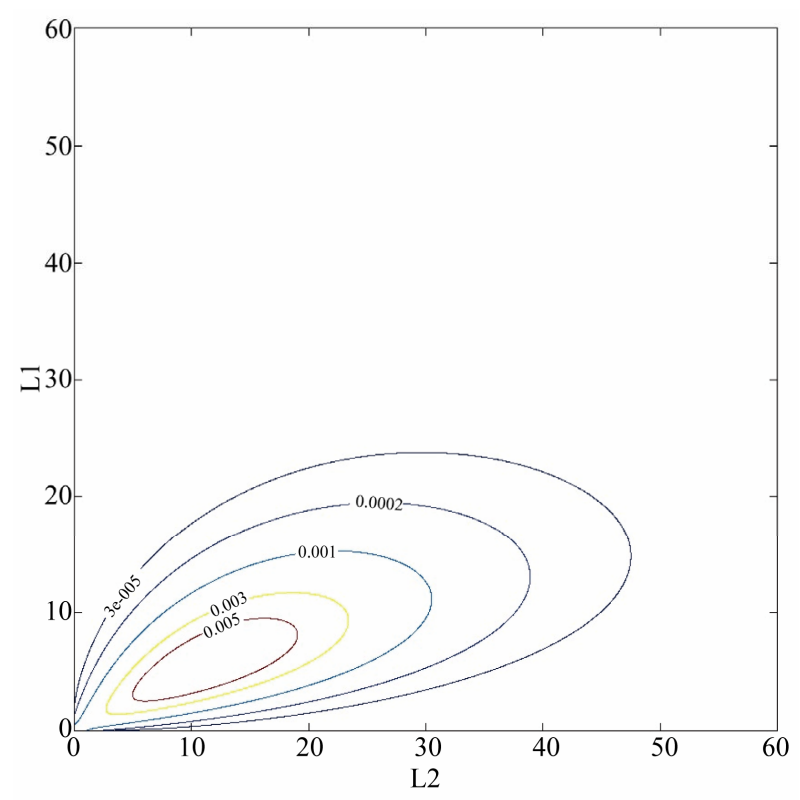

Figure 6. The contour of the joint distribution of collateral losses with $\theta=1$ for a Poisson process. 


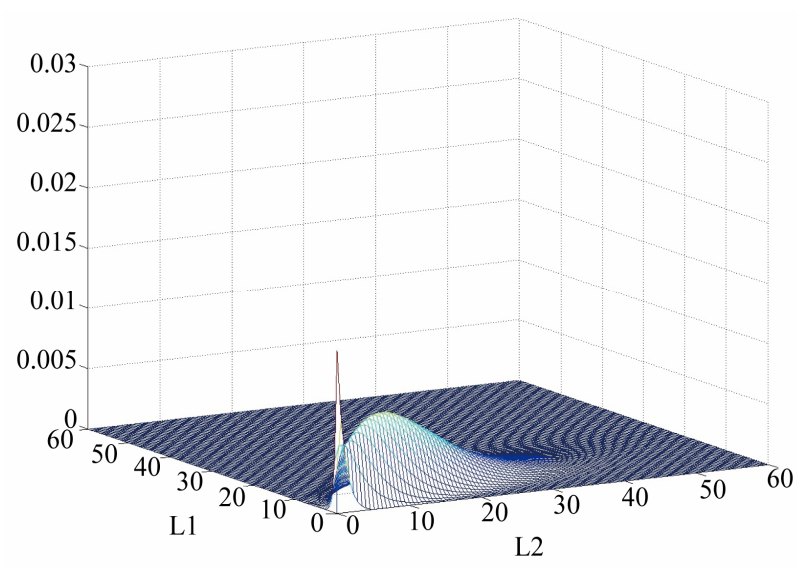

Figure 7. The joint distribution of collateral losses with $\theta=$ 1 and $\rho=4$.

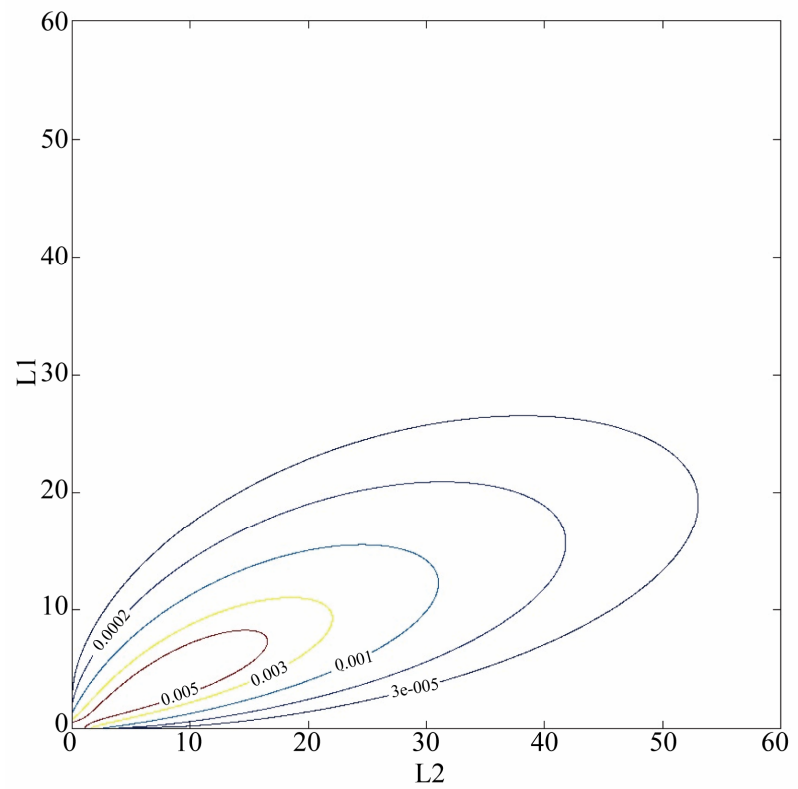

Figure 8. The contour of the joint distribution of collateral losses with $\theta=1$ and $\rho=4$.

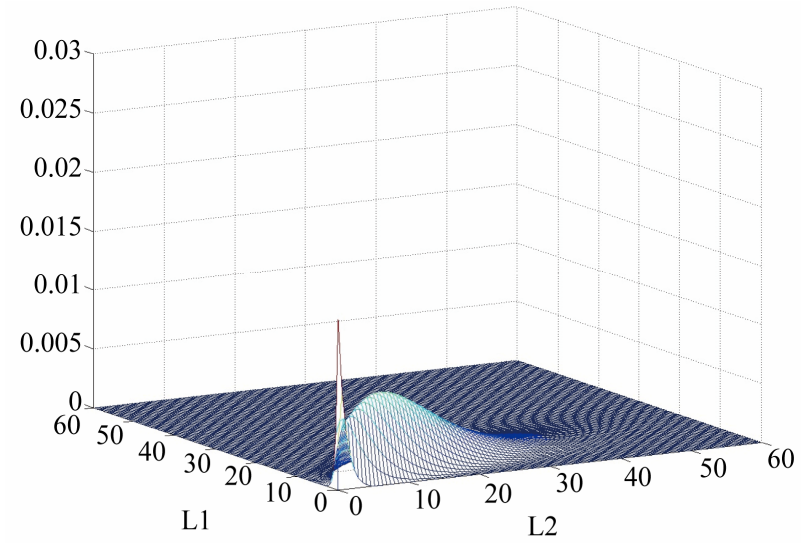

Figure 9. The joint distribution of collateral losses with $\theta=$ 1 and $\delta=0.25$.

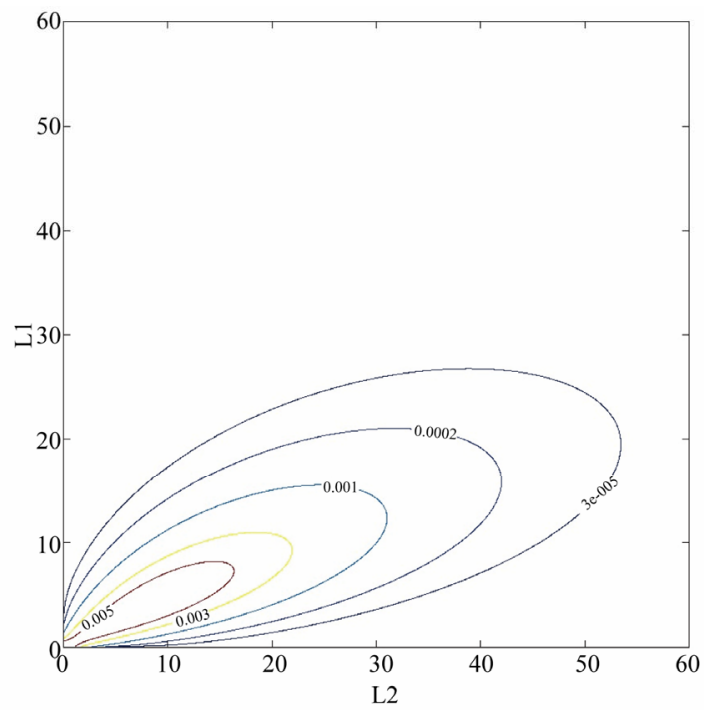

Figure 10. The contour of the joint distribution of collateral losses with $\theta=1$ and $\delta=0.25$.

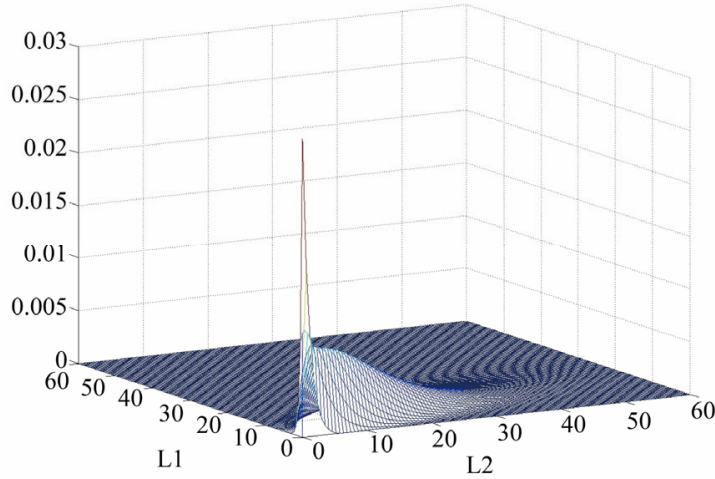

Figure 11. The joint distribution of collateral losses with $\theta=$ 1 and $\gamma=0.5$.

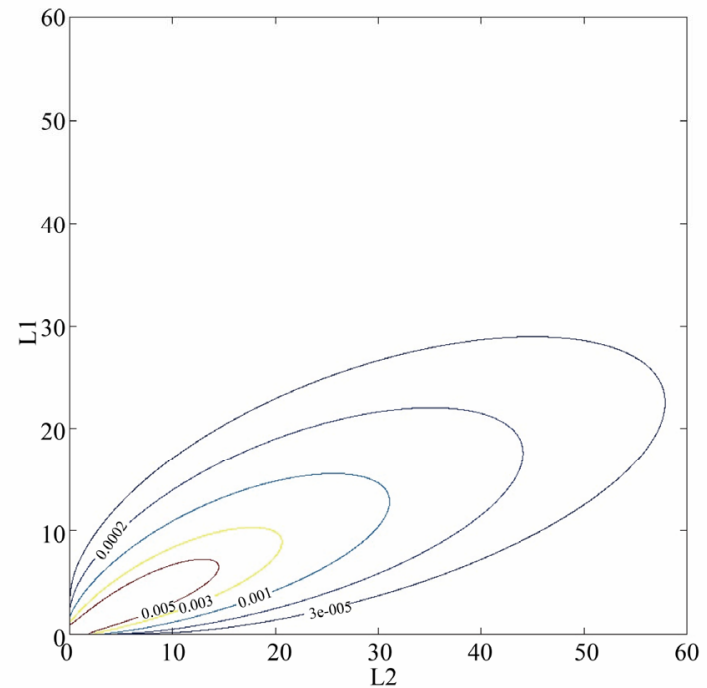

Figure 12. The contour of the joint distribution of collateral losses with $\theta=1$ and $\gamma=0.5$. 
Table 19. $\mathbb{E}\left\{\boldsymbol{L}_{t}^{(1)} \mid \boldsymbol{L}_{t}^{(1)}+\boldsymbol{L}_{t}^{(2)}>\boldsymbol{F}_{L_{t}^{(1)}+L_{t}^{(2)}}^{-(u)}\right\}$

\begin{tabular}{ccccc}
\hline$\theta=1 \quad q \%$ & A Poisson process & \multicolumn{3}{c}{ A shot-noise Cox process } \\
\hline & & $\rho=4$ & $\delta=0.25$ & $\gamma=0.5$ \\
\hline $95 \%$ & 18.52079 & 21.41422 & 21.37291 & 23.50179 \\
$99 \%$ & 22.33392 & 26.15472 & 26.47892 & 29.68759 \\
\hline
\end{tabular}

tours at $\theta=1$ with respect to a shot-noise Cox process for $N_{t}$.

Figures 7-12 (shot-noise Cox cases) show how different joint probabilities of aggregate collateral losses are from Figures 5-6 (a Poisson case) by changes in the value of $\rho, \delta$ and $\gamma$, respectively. Even though they have the same mean, the joint probabilities of aggregate collateral losses for shot-noise Cox cases are located more between the bottom left corner and the top right corner when $\theta=1$ than its counterpart. Shot-noise Cox cases display that they have heavier tail than their counterparts, which are shown in Table 19 in terms of the risk measure of (4). Compared to the effect on the risk measure of (4) by changes in the value of $\rho, \delta$ and $\gamma$, respectively from a Poisson case, Table 19 shows that $\gamma$, which is the parameter in exponential jump size distribution of catastrophic event, is the most sensitive parameter in terms of changes in the value the risk measure of (4).

\section{Conclusions}

We have used bivariate compound process to model aggregate collateral losses arising from catastrophic events such as flood, storm, hail, bushfire and earthquake. For the number of collateral losses, a Cox process was used to accommodate the stochastic nature of their frequency rate in practice. The shot noise process was used as the intensity of a Cox process as the number of collateral losses arising from catastrophic events depends on the frequency and magnitude of the primary events and the time period needed to determine the effect of these events. We also examined a Poisson process for the number of collateral losses as its counterpart. With the common collateral loss arrival process in the bivariate model, the dependence between the individual losses arising from different risk type has been modelled using the notion of copula where for numerical illustrations, a member of Farlie-Gumbel-Morgenstern copula with exponential margins was used.

As it was difficult to derive the joint distributions the aggregate collateral losses, we derived their Laplace transforms and inverted their Fast Fourier transforms numerically to calculate introduced relevant risk measures. These measures can be used to calculate tail dependences between collateral losses or to calculate in- surance premiums. We have presented the expressions for joint probabilities of the aggregate collateral losses and their densities at $L_{t}^{(1)}=0$ and $L_{t}^{(2)}=0$, which were used to improve the accuracy of joint distributions of the aggregate collateral losses inverting the Fast Fourier transforms. We have compared the simulated risk measure values obtained using a compound Poisson and a compound shot-noise Cox model, respectively. The sensitivity analysis on the parameters of a shot-noise Cox process from a Poisson process also provided using the risk measure of (4).

Other counting processes and other copulas with different margins can be considered in the proposed bivariate model, that we leave for further research. We hope that what we have presented in this paper provides practitioners with feasible models to quantify collateral losses that would occur more often due to global warming, climate change and terrorism.

\section{REFERENCES}

[1] N. Bäuerle and A. Müller, "Modeling and Comparing Dependencies in Multivariate Risk Portfolios," ASTIN Bulletin, Vol. 28, No. 1, 1998, pp. 59-76. doi:10.2143/AST.28.1.519079

[2] H. Cossette, P. Gaillardetz, E. Marceau and J. Rioux, "On Two Dependent Individual Risk Models," Insurance: Mathematics and Economics, Vol. 30, No. 2, 2002, pp. 153-166. doi:10.1016/S0167-6687(02)00094-X

[3] C. Genest, E. Marceau and M. Mesfioui, "Compound Poisson Approximations for Individual Models with Dependent Risks," Insurance: Mathematics and Economics, Vol. 32, No. 1, 2003, pp. 73-91. doi:10.1016/S0167-6687(02)00205-6

[4] N. Bäuerle and R. Grübel, "Multivariate Counting Processes: Copulas and Beyond," ASTIN Bulletin, Vol. 35, No. 2, 2005, pp. 379-408. doi:10.2143/AST.35.2.2003459

[5] M. L. Centeno, "Dependent Risks and Excess of Loss Reinsurance," Insurance: Mathematics and Economics, Vol. 37, No. 2, 2005, pp. 229-238. doi:10.1016/j.insmatheco.2004.12.001

[6] F. Lindskog and A. J. McNeil, "Common Poisson Shock Models: Applications to Insurance and Credit Risk Modelling," ASTIN Bulletin, Vol. 33, No. 2, 2003, pp. 209238. doi:10.2143/AST.33.2.503691

[7] D. Pfeifer and J. Nešlehová, "Modeling and Generating Dependent Risk Processes for IRM and DFA," ASTIN 
Bulletin, Vol. 34, No. 2, 2004, pp. 333-360. doi:10.2143/AST.34.2.505147

[8] V. Chavez-Demoulin, P. Embrechts and J. Nešlehová, "Quantitative Models for Operational Risk: Extremes, Dependence and Aggregation," Journal of Banking and Finance, Vol. 30, No. 10, 2006, pp. 2635-2658. doi:10.1016/j.jbankfin.2005.11.008

[9] R. Cont and P. Tankov, "Financial Modelling with Jump Processes," Champan \& Hall, Boca Raton, 2004.

[10] Victorian Bushfires Royal Commission, "Final ReportSummary, Parliament of Victoria," Parliament of Victoria, Melbourne, 2010.

[11] M. L. Burton and M. J. Hicks, "Hurricane Katrina: Preliminary Estimates of Commercial and Public Sector Damages," Marshall University, Huntington, 2005.

[12] G. Makinen, "The Economic Effects of 9/11: A Retrospective Assessment," Congressional Research Service, Washington DC, 2002.

[13] R. B. Nelsen, "An Introduction to Copulas," SpringerVerlag, New York, 1999.

[14] B. Schweizer and A. Sklar, "Probabilistic Metric Spaces," Elsevier, New York, 1983.

[15] C. Klüppelberg and T. Mikosch, "Explosive Poisson Shot Noise Processes with Applications to Risk Reserves," Bernoulli, Vol. 1, No. 1-2, 1995, pp. 125-147. doi: $10.2307 / 3318683$

[16] A. Dassios and J. Jang, "Pricing of Catastrophe Reinsurance \& Derivatives Using the Cox Process with Shot Noise Intensity," Finance \& Stochastics, Vol. 7, No. 1, 2003, pp. 73-95. doi:10.1007/s007800200079

[17] A. Dassios and J. Jang, "Kalman-Bucy Filtering for Linear System Driven by the Cox Process with Shot Noise Intensity and Its Application to the Pricing of Reinsurance Contracts," Journal of Applied Probability, Vol. 42, No. 1, 2005, pp. 93-107. doi:10.1239/jap/1110381373

[18] A. Dassios and J. Jang, "The Distribution of the Interval between Events of a Cox Process with Shot Noise Intensity," Journal of Applied Mathematics and Stochastic Analysis, Vol. 2008, 2008, Article ID: 367170. doi: $10.1155 / 2008 / 367170$

[19] J. Jang and Y. Krvavych, "Arbitrage-Free Premium Calculation for Extreme Losses Using the Shot Noise Process and the Esscher Transform," Insurance: Mathematics and Economics, Vol. 35, No. 1, 2004, pp. 97-111. doi:10.1016/j.insmatheco.2004.05.002

[20] J. Jang and G. Fu, "Transform Approach for Operational Risk Management: VaR and TCE," Journal of Operational Risk, Vol. 3, No. 2, 2008, pp. 45-61.

[21] D. R. Cox and V. Isham, "Point Processes," Chapman \& Hall, London, 1980.

[22] A. McNeil, R. Frey and P. Embrechts, "Quantitative Risk Management: Concepts, Techniques and Tools," Princeton University Press, Princeton, 2005.

[23] M. H. A. Davis, "Piecewise Deterministic Markov Processes: A General Class of Non Diffusion Stochastic Models," Journal of the Royal Statistical Society Series B, Vol. 46, No. 3, 1984, pp. 353-388.

[24] A. Dassios and P. Embrechts, "Martingales and Insurance Risk," Communications in Statistics. Stochastic Models, Vol. 5, No. 2, 1989, pp. 181-217. doi:10.1080/15326348908807105

[25] T. Rolski H. Schmidli, V. Schmidt and J. L. Teugels, "Stochastic Processes for Insurance and Finance," John Wiley \& Sons, Hoboken, 1998.

[26] K. R. Castleman, "Digital Image Processing," Prentice Hall, Englewood Cliffs, 1996.

[27] R. C. Gonzalez and R. E. Woods, "Digital Image Processing," 2nd Edition, Prentice Hall, Upper Saddle River, 2002.

[28] R. C. Gonzalez, R. E. Woods and S. L. Eddins, "Digital Image Processing Using MATLAB," Prentice Hall, Upper Saddle River, 2004. 